\title{
Effect of the surface magnetic anisotropy of neodymium atoms on the coercivity in neodymium permanent magnets
}

\author{
Masamichi Nishino $\odot,{ }^{1,2, *}$ Ismail Enes Uysal $\odot,{ }^{2}$ and Seiji Miyashita ${ }^{3,4,5,2}$ \\ ${ }^{1}$ Research Center for Advanced Measurement and Characterization, National Institute for Materials Science, \\ 1-2-1 Sengen, Tsukuba, Ibaraki 305-0047, Japan \\ ${ }^{2}$ Elements Strategy Initiative Center for Magnetic Materials, National Institute for Materials Science, \\ 1-2-1 Sengen, Tsukuba, Ibaraki 305-0047, Japan \\ ${ }^{3}$ Department of Physics, Graduate School of Science, The University of Tokyo, 7-3-1 Hongo, Tokyo 113-0033, Japan \\ ${ }^{4}$ The Physical Society of Japan, 2-31-22 Yushima, Tokyo 113-0033, Japan \\ ${ }^{5}$ Institute for Solid State Physics, The University of Tokyo, 5-1-5 Kashiwanoha, Kashiwa 277-8581, Japan
}

(Received 20 August 2020; accepted 22 December 2020; published 13 January 2021)

\begin{abstract}
The Nd permanent magnet $\left(\mathrm{Nd}_{2} \mathrm{Fe}_{14} \mathrm{~B}\right)$ is an indispensable material used in modern energy conversion devices. The realization of high coercivity at finite temperatures is an important issue. One of the important ingredients for controlling the coercive force is the surface property of magnetic grains. It has been found by first-principles studies that the $\mathrm{Nd}$ atoms in the first (001) surface layer facing the vacuum have in-plane anisotropy perpendicular to the $c$ axis, which may decrease the coercivity. Focusing on the surface anisotropy effect on the coercivity, we examine the coercivity at zero and finite temperatures using an atomistic model reflecting the lattice structure of the Nd magnet with a stochastic Landau-Lifshitz-Gilbert equation method. We study three general cases, in which the Nd atoms in surface layers have (1) no anisotropy, (2) in-plane anisotropy, and (3) reinforced anisotropy for two types of surface, (001) and (100) surfaces. We find that in contrast to the zero-temperature case, owing to the thermal fluctuation effect, the modification of only the first surface layer has little effect on the coercivity at finite temperatures. However, the modification of a few layers results in significant effects. We discuss the details of the dependences of the coercivity on temperature, the type of surface, and modified layer depth, and also the features of domain growth in magnetization reversal.
\end{abstract}

DOI: 10.1103/PhysRevB.103.014418

\section{INTRODUCTION}

The realization of stronger coercivity at higher temperatures is a central issue in the development of permanent magnet materials for obtaining higher efficiency in energy conversion devices such as electric motors, generators, and electronic devices [1]. The $\mathrm{Nd}$ magnet, $\mathrm{Nd}_{2} \mathrm{Fe}_{14} \mathrm{~B}$, is a very important target magnet because of its high coercive force [2-10].

Its coercivity depends on several factors, e.g., magnetic properties of grains (hard magnet phase) and grain boundaries (soft magnet phase) [11-18]. Surface nucleation at the hard magnet is an essential process for magnetization reversal. Thus, the property of the surfaces of grains is a very important factor of the coercivity [19].

Micromagnetic continuum modeling for permanent magnets has been applied in analyses of magnetic properties of the $\mathrm{Nd}$ magnet [20]. However, to study the microscopic details, recently developed atomistic models are indispensable [16,21-33]. Unlike in continuum modeling, the lattice structure (Fig. 1) is introduced with microscopic magnetic parameters and the temperature effect can be treated properly in atomistic modeling. Quantitative properties of the Nd magnet

*Corresponding author: nishino.masamichi@nims.go.jp have been intensively investigated and important properties have been clarified using atomistic models [16,21-33].

The temperature dependence of magnetization has been reproduced and it catches the characteristic spin-reorientation (SR) transition of the Nd magnet [21-24,29]. The domain wall profiles have been shown as a function of temperature and the estimated domain wall widths are in good agreement with experimental data $[22,24,25,29,30]$. From a dynamical aspect, the non-monotonic temperature dependence of the ferromagnetic resonance originating from the SR transition has been shown [26].

The crystal (amorphas) structures of grain boundary phases are complicated and have not been clarified yet. Trials of the estimation of those structures by first-principles calculations have just begun [34,35]. In the current stage of atomistic modeling, some assumptions for the structures, magnetic parameters, and so forth are necessary for the grain boundary phases. Very recently, the coercive force, which is a long-time phenomenon, i.e., $1 \mathrm{~s}$, for an isolated single grain was estimated to be around $3 \mathrm{~T}$ by calculating the free-energy barrier [27] and also by a direct simulation of dynamics [28]. Inhomogeneity effects due to grain boundaries on the coercivity have also been studied [16,25,30-33], and characteristics of the nucleation and pinning fields have been shown as functions of magnetic parameters of the soft magnet phase (grain boundary) [16]. 
(a)

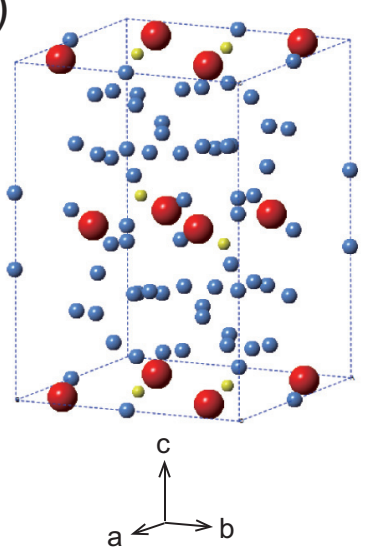

(b)

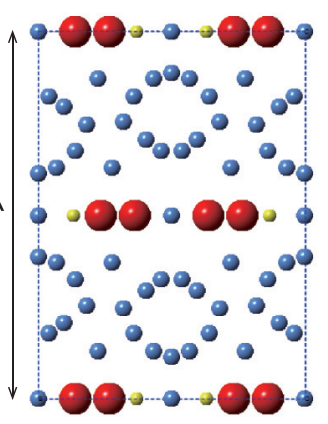

(c)

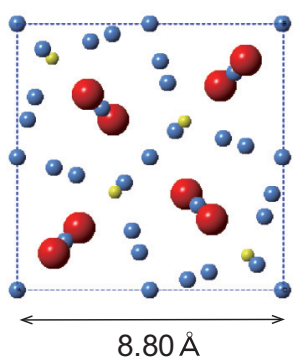

FIG. 1. (a) Unit cell of $\mathrm{Nd}_{2} \mathrm{Fe}_{14} \mathrm{~B}$. Neodymium, iron, and boron atoms are denoted by red, blue, and yellow spheres, respectively. The lattice constants [3] for the $a, b$, and $c$ axes are $d_{\mathrm{a}}=d_{\mathrm{b}}=8.80 \AA$ and $d_{\mathrm{c}}=12.19 \AA$, respectively. (b) Side view (from $a$ or $b$ axis). (c) Top view (from $c$ axis).

The anisotropy energy for a rare-earth atom (ion) in the crystal electric field (CEF) is given by the following Hamiltonian:

$$
\mathcal{H}_{\mathrm{CEF}}=\sum_{l, m} B_{l}^{m} \hat{O}_{l}^{m},
$$

where $B_{l}^{m}=\Theta_{l} A_{l}^{m}\left\langle r^{l}\right\rangle$. Here, $B_{l}^{m}$ is the CEF coefficient and $\hat{O}_{l}^{m}$ is the Stevens operator, e.g., $\hat{O}_{2}^{0}=3 J_{z}^{2}-J^{2}$, where $J=$ 9/2 for Nd atoms. $\Theta_{l}, A_{l}^{m}$, and $\left\langle r^{l}\right\rangle$ are the Stevens factor, the coefficient of the spherical harmonics of the crystalline electric field, and the average of $r^{l}$ over the radial wave function, respectively.

The Nd magnet shows the SR transition at $T_{\mathrm{R}} \sim 150 \mathrm{~K}$, in which the magnetization is tilted from the $c$ axis at $0 \mathrm{~K}$ and becomes parallel to the $c$ axis above $T_{\mathrm{R}}$.

Substituting $J_{z}=J \cos \theta$ into Eq. (1), the anisotropy energy at $0 \mathrm{~K}$ for $\mathrm{Nd}$ atoms is expressed with diagonal terms in the following form:

$$
E_{\mathrm{A}}=K_{1} \sin ^{2} \theta+K_{2} \sin ^{4} \theta+K_{4} \sin ^{6} \theta,
$$

where

$$
K_{1}=-3 f_{2} B_{2}^{0}-40 f_{4} B_{4}^{0}-168 f_{6} B_{6}^{0}
$$

with constants $f_{l}(>0)$. The $\mathrm{SR}$ transition at $T_{\mathrm{R}}$ is well reproduced by the coefficients $A_{0}^{2}=295.0 \mathrm{~K} a_{0}^{-2}, A_{0}^{4}=-12.3 \mathrm{~K}$ $a_{0}^{-4}$, and $A_{0}^{6}=-1.84 \mathrm{~K} a_{0}^{-6}$ estimated by Yamada et al. [36], in which $a_{0}$ is the Bohr radius. Although $A_{0}^{2}>0$ and $B_{2}^{0}<0$ $\left(\because \Theta_{2}<0\right)$, the first single-ion anisotropy $K_{1}<0$ owing to the contribution of the other terms ( $B_{4}^{0}$ and $B_{6}^{0}$ terms). This causes a tilted magnetization in the ground state (at zero tem-

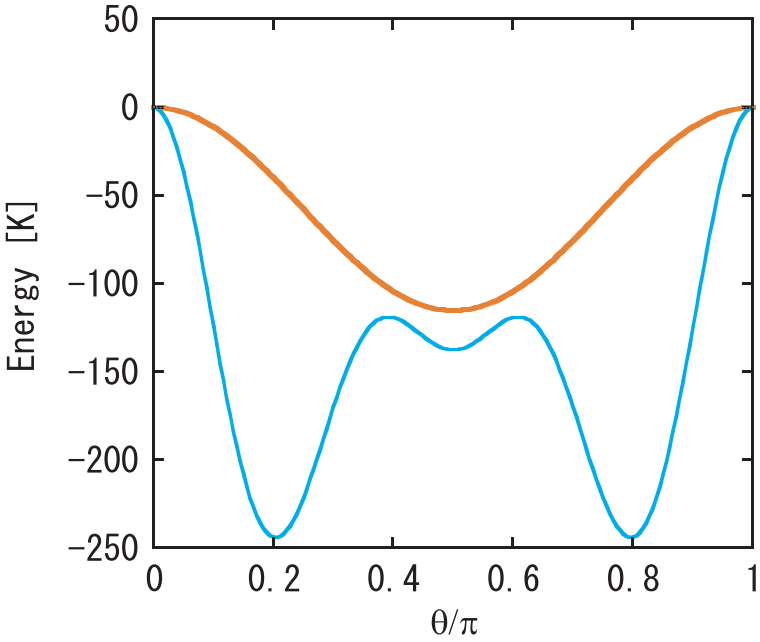

FIG. 2. Anisotropy potential of the Nd atom (blue line) and inplane anisotropy potential (red line) used in case (2) as functions of the angle $\theta$ from the $c$ axis.

perature), as shown in Fig. 2 (blue line), in which $\theta \simeq 0.2 \pi$ gives the minimum.

At finite temperatures, the moment $J_{z}$ shrinks owing to the thermal fluctuation as $J_{z}=C J \cos \theta$ with $0<C<1$. The coefficient $C$ is estimated to be $C \simeq 0.76$ at $T=0.46 T_{\mathrm{c}}\left(T_{\mathrm{c}}\right.$ is the Curie temperature) close to room temperature in the bulk from the temperature dependence of the magnetization in our previous study [22]. Thus, substituting $J_{z}=0.76 \mathrm{~J} \cos \theta$ into Eq. (1), the effective $K_{1}(T)$ in the form of Eq. (2) is found to be $K_{1}(T)>0$. Thus, for $T>T_{\mathrm{R}}$, the bottleneck structure at $0 \mathrm{~K}$ is smeared out and a minimum at $\theta=0$ is realized, as shown in Fig. 3 (blue line).

First-principles studies based on density functional theory reported that the $\mathrm{Nd}$ atoms in the first (001) surface layer have in-plane anisotropy perpendicular to the $c$ axis, different from out-of-plane anisotropy in the bulk, and this may reduce coercivity $[37,38]$. Those studies showed $A_{0}^{2}<0$ or $B_{0}^{2}>0$, for the $\mathrm{Nd}$ atoms at the (001) surface, which is different from that

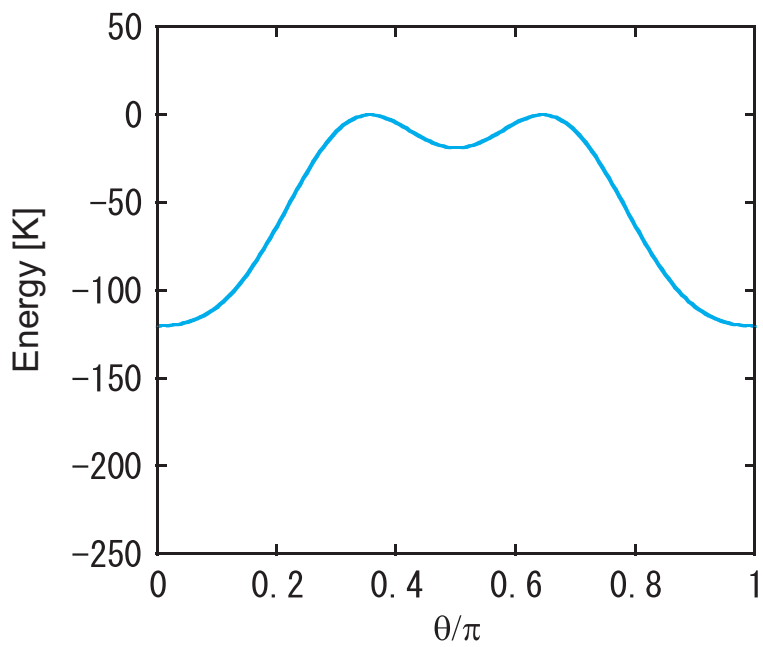

FIG. 3. Effective anisotropy potential of the $\mathrm{Nd}$ atom at $T=$ $0.46 T_{\mathrm{c}}$ as a function of the angle $\theta$ from the $c$ axis. 
(a)

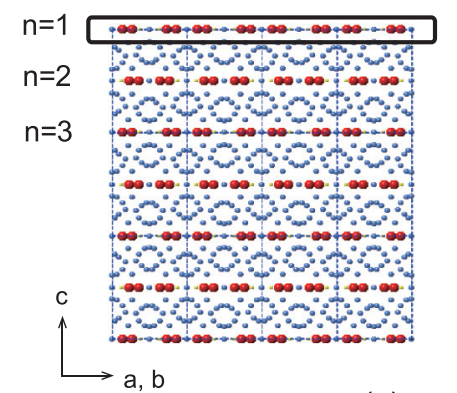

(c)

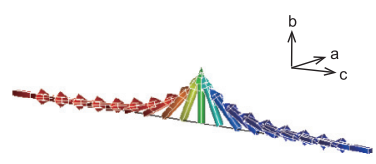

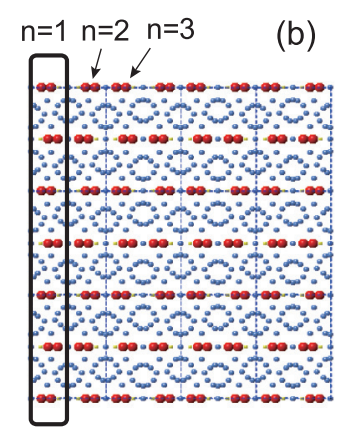

(d)

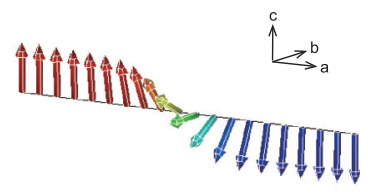

FIG. 4. Definition of the Nd layer number for the (a) (001) and (b) (100) surfaces. (c) Néel domain wall moving along the $c$ axis. (d) Bloch domain wall moving along the $a$ axis.

in the bulk, i.e., $A_{0}^{2}>0$ or $B_{0}^{2}<0$, and the strength of $A_{0}^{2}$ at the surface was comparable to that in the bulk $[37,38]$. Although the conclusions of those studies were derived within the firstterm approximation, i.e., $K_{1} \sim-3 f_{2} B_{2}^{0}$ (namely, $A_{0}^{4}=0$ and $A_{0}^{6}=0$ ), it indicates that the anisotropy at the surface is of the in-plane type.

A large reduction in coercive field was found at zero temperature by a Landau-Lifshitz-Gilbert (LLG) simulation taking into account the in-plane anisotropy at the surface in a simplified lattice (cubic lattice) model in a previous study [39]. However, in that study, the effect of a tilted structure of the ground state was not considered. Furthermore, the temperature effect, which may change the situation, has not been examined. Thus, in the present paper, we study the surface magnetic anisotropy effect of $\mathrm{Nd}$ atoms in the $\mathrm{Nd}_{2} \mathrm{Fe}_{14} \mathrm{~B}$ magnet on the coercive force using a recently developed atomistic model constructed on the basis of the real crystal structure (Fig. 1). We investigate the temperature dependence of the effects of not only the (001) surface but also the (100) surface by employing the stochastic LLG (SLLG) equation $[40,41]$. We adopt $A_{0}^{2}, A_{0}^{4}$, and $A_{0}^{6}$ given by Yamada et al. for the atomistic model in bulk cells (see Sec. II and Fig. 4).

Experimental trials of increasing the coercivity by replacing surface $\mathrm{Nd}$ atoms by other rare-earth atoms have been performed. The coercive field of the Nd magnet has been increased by adding Dy atoms. It has been recently reported that an annealing process after the diffusion formed Dy-rich second shells with widths of a few nanometers, and those shells increase the coercive force by 30 to $40 \%$ [42]. The mechanism underlying this increase is an open question. A reinforcement of the anisotropy energy of surface rare-earth atoms may be one of the keys to the increase in coercivity. In the present study, we focus on three typical cases for the surface anisotropy effect: (1) no anisotropy, (2) in-plane anisotropy, and (3) reinforced anisotropy for surface $\mathrm{Nd}$ atoms.

The rest of the paper is organized as follows. In Sec. II, the atomistic Hamiltonian, systems with different surface

anisotropies, and the dynamical method are shown. In Sec. III, the coercive fields in the three cases for the (001) and (100) surfaces at zero and finite temperatures are presented. Discussions about the surface anisotropy effects on the coercivity are also given. In Sec. IV, features of domain growth are discussed. Section V is devoted to the summary.

\section{MODEL AND METHOD}

\section{A. Atomistic Hamiltonian for $\mathrm{Nd}_{2} \mathrm{Fe}_{14} \mathrm{~B}$}

We adopt the following atomistic Hamiltonian for the $\mathrm{Nd}$ magnet [21-23]:

$$
\begin{aligned}
\mathcal{H}= & -\sum_{i<j} 2 J_{i j} s_{i} \cdot s_{j}-\sum_{i}^{\mathrm{Fe}} D_{i}\left(s_{i}^{z}\right)^{2} \\
& +\sum_{i}^{\mathrm{Nd}} \sum_{l, m} \Theta_{l, i} A_{l, i}^{m}\left\langle r^{l}\right\rangle_{i} \hat{O}_{l, i}^{m}-h \sum_{i} S_{i}^{z} .
\end{aligned}
$$

Here, $J_{i j}$ is the exchange interaction between the $i$ th and $j$ th sites. Fe atoms have small magnetocrystalline anisotropy energies and $D_{i}$ is the anisotropy constant of $\mathrm{Fe}$ atoms. The third term is the CEF (1) for the magnetic anisotropy energy of $\mathrm{Nd}$ atoms, and $h$ is the external magnetic field applied to the $i$ th site. The crystal structure of the unit cell is shown in Fig. 1(a). For Fe and B atoms, $s_{i}$ denotes the magnetic moment at the $i$ th site, but for $\mathrm{Nd}$ atoms, it is the moment of valence $(5 d$ and $6 s$ ) electrons, and it is coupled antiparallel to the moment of 4- $f$ electrons, $\mathcal{J}_{i}$. Thus, the total moment for each $\mathrm{Nd}$ atom is $\boldsymbol{S}_{i}=\boldsymbol{s}_{i}+\mathcal{J}_{i}$. Here, $\mathcal{J}_{i}=g_{\mathrm{T}} J \mu_{\mathrm{B}}$, in which $g_{\mathrm{T}}=8 / 11$ is the Lande $\mathrm{g}$ factor and $J=9 / 2$ is the magnitude of the total angular momentum. For the $\mathrm{Fe}$ and $\mathrm{B}$ atoms, we define $S_{i}=s_{i}$. In the third term the summation for $l$ runs $l=2,4$, and 6 , and we consider only the diagonal operators $(m=0)$, which give dominant contributions.

We use the same parameter values for the atomistic model as in our previous studies [21-23], in which the exchange interactions within the range of $r=3.52 \AA$ for each atom were estimated by first-principles calculation with the KorringaKohn-Rostoker Green's function method [43], $D_{i}$ values for $\mathrm{Fe}$ atoms (six kinds) estimated in a first-principles study [44] were adopted, and for $\mathrm{Nd}$ atoms $A_{l}^{m}$ given by Yamada et al. [36] were adopted with $\left\langle r^{l}\right\rangle$ estimated in Ref. [45]. Employing these parameters, we showed the SR transition at the transition temperature $T_{\mathrm{R}}=150 \mathrm{~K}$, which is close to experimentally estimated ones [8-10,36], and the critical temperature $T_{\mathrm{c}} \sim$ $850 \mathrm{~K}[21,22]$, which is slightly overestimated compared with the experimental values of $T_{\mathrm{c}} \sim 600 \mathrm{~K}[5,8]$.

\section{B. Systems}

Throughout this paper, we study a system with $12 \times 12 \times$ 9 unit cells along the $a, b$, and $c$ axes, respectively $(10.56 \mathrm{~nm}$ $\times 10.56 \mathrm{~nm} \times 10.971 \mathrm{~nm})$. We consider two types of surface, i.e., (001) and (100) surfaces, as depicted in Fig. 4. The $\mathrm{Nd}$ layers at the (001) surface are numbered as in Fig. 4(a). Nd atoms in the (100) surface at $n=1,2, \cdots$ are defined as those located between $a=0$ and $a=d_{\mathrm{a}} / 2$, between $a=d_{\mathrm{a}} / 2$ and $a=d_{\mathrm{a}}, \ldots$, where $d_{\mathrm{a}}$ is the lattice constant of the $a$ axis [Fig. 4(b)]. Open and periodic boundary conditions are 
used along the $c$ axis and the $a$ and $b$ axes, respectively, for the (001) surface, whereas open and periodic boundary conditions are used along the $a$ axis and the $b$ and $c$ axes, respectively, for the (100) surface. Thus, in each system, two surface planes in contact with vacuum exist. A Néel-type domain wall (DW) moves in the system with the (001) surface [Fig. 4(c)], whereas a Bloch-type DW moves in that with the (100) surface [Fig. 4(d)].

We investigate the coercive force in the following three cases of anisotropy parameters $\left(\tilde{A}_{2}^{0}, \tilde{A}_{4}^{0}, \tilde{A}_{6}^{0}\right)$ for the surface $\mathrm{Nd}$ atoms.

Case (1) No anisotropy in $\mathrm{Nd}$ atoms:

$$
\tilde{A}_{2}^{0}=\tilde{A}_{4}^{0}=\tilde{A}_{6}^{0}=0 .
$$

Case (2) In-plane anisotropy in $\mathrm{Nd}$ atoms:

$$
\tilde{A}_{2}^{0}=-A_{2}^{0}<0, \quad \text { and } \quad \tilde{A}_{4}^{0}=\tilde{A}_{6}^{0}=0,
$$

where $\tilde{A}_{0}^{2}$ is negative and the amplitude is on the same order as that in the bulk $\left(A_{0}^{2}=295.0 \mathrm{Ka}_{0}^{-2}\right)$.

Case (3) Doubly reinforced anisotropy in Nd atoms:

$$
\tilde{A}_{2}^{0}=2 A_{2}^{0}, \quad \tilde{A}_{4}^{0}=2 A_{4}^{0}, \quad \text { and } \quad \tilde{A}_{6}^{0}=2 A_{6}^{0} .
$$

\section{Dynamical method}

We study threshold fields under a reversed field using the SLLG equation $[40,41]$,

$$
\frac{d}{d t} \boldsymbol{S}_{i}=-\frac{\gamma}{1+\alpha_{i}^{2}} \boldsymbol{S}_{i} \times \boldsymbol{h}_{i}^{\mathrm{eff}}-\frac{\alpha_{i} \gamma}{\left(1+\alpha_{i}^{2}\right) S_{i}} \boldsymbol{S}_{i} \times\left[\boldsymbol{S}_{i} \times \boldsymbol{h}_{i}^{\mathrm{eff}}\right] .
$$

Here, $\gamma$ denotes the electron gyromagnetic ratio and $\alpha_{i}$ is the damping parameter.

The effective field $\boldsymbol{h}_{i}^{\text {eff }}$ on the $i$ th spin consists of the exchange interaction field, the anisotropy field, the external field, and a noise field for thermal fluctuation, and it is given as

$$
\boldsymbol{h}_{i}^{\mathrm{eff}}=-\frac{\partial \mathcal{H}}{\partial \boldsymbol{S}_{i}}+\boldsymbol{\xi}_{i}(t) \text {. }
$$

Here, the noise field $\boldsymbol{\xi}_{i}(t)=\left(\xi_{i}^{x}, \xi_{i}^{y}, \xi_{i}^{z}\right)$ is of white Gaussian and satisfies the following relations:

$$
\left\langle\xi_{i}^{\mu}(t)\right\rangle=0, \quad\left\langle\xi_{i}^{\mu}(t) \xi_{j}^{\nu}(s)\right\rangle=2 \mathcal{D}_{i} \delta_{i j} \delta_{\mu \nu} \delta(t-s) .
$$

The temperature $T$ is given by a function of the amplitude of the noise $\mathcal{D}_{i}$ according to the fluctuation dissipation relation

$$
\mathcal{D}_{i}=\frac{\alpha_{i} k_{\mathrm{B}} T}{\gamma S_{i}} .
$$

With this relation, the system relaxes to a steady state (equilibrium) in the canonical distribution of the temperature $T$.

Simulations were performed by solving Eq. (8) numerically. The damping parameter is unknown for the $\mathrm{Nd}$ magnet, and $\alpha_{i}$ is set to 0.1 . This choice does not affect the results in the present study because $\alpha_{i}$ slightly affects the threshold field [28]. We employ a kind of middle-point method [41] equivalent to the Heun method for numerical integration. For the time step of this equation, $\Delta t=0.1 \mathrm{fs}$ is used.
Starting from a down-spin state for all spins, we calculated the time evolution of magnetization

$$
M_{z}=\sum_{i} S_{i, z}
$$

under a given value of magnetic field $h$ in the $c$ direction. We define the time of reversal as the time when the magnetization changes its sign. At zero temperature, there is no thermal fluctuation, and thus when the magnetic field reaches the value at which the potential barrier vanishes, this value gives the threshold field for magnetization reversal, i.e., coercive field. This process is deterministic. We regarded the final state as the state when all the motion stops. In the simulation, the final state was obtained within $N_{t}=1 \times 10^{6}$ time steps $(t=0.1 \mathrm{~ns})$.

In contrast to the zero-temperature reversal, at finite temperatures the magnetization reversal exhibits a stochastic process to jump over the energy barrier by thermal fluctuation. We defined the threshold magnetic field at finite temperatures as follows. We performed 12 simulations with different random number sequences. At each value of the magnetic field, we counted the number of cases, $N$, in which magnetization reversal took place within a simulation time $t_{\max }$. If $N=0$, the field is smaller than the threshold field, whereas if $N=12$, the field is larger than the threshold field. We defined the threshold field as the middle point of the interval between the field of $N=0$ and that of $N=12$. The error bar for the threshold field was defined as the transient region of the field. The threshold field depends on the simulation time. Here, we set the simulation time to $t_{\max }=0.5 \mathrm{~ns}\left(5 \times 10^{6}\right.$ time steps). The measurement time for the coercivity should be on the order of $1 \mathrm{~s}$ in experiments, but this time scale is not practical for real-time simulations. However, the dependence of the reversal time on the external field around the threshold field is very sharp, i.e., the reversal time exponentially increases. In our previous paper [28], we estimated that the threshold field for $1 \mathrm{~s}$ is about $25 \%$ less than that of the simulation for $0.5 \mathrm{~ns}$. Thus, we expect that the estimated threshold fields can give approximate coercive fields.

\section{THRESHOLD FIELDS FOR MAGNETIZATION REVERSAL}

\section{A. Zero-temperature properties}

First, we study the threshold field at $T=0 \mathrm{~K}$. In Figs. 5(a) and 5(b), we depict how the threshold field depends on the number of modified layers, $n$, in cases (1), (2), and (3) for both the (001) and (100) surfaces, respectively. Here, $n=0$ is defined as the case without modification of the anisotropy of surface $\mathrm{Nd}$ atoms, i.e., the anisotropy of the surface $\mathrm{Nd}$ atoms is the same as that in the bulk. The value (percentage) given for each symbol in the figures denotes the ratio of the threshold field to that for $n=0$.

We find that for both surfaces, the modification of the one-layer surface $(n=1)$ markedly decreases the threshold field by around $25 \%$ in cases (1) and (2), and the field decreases further for larger $n$. This indicates that at $T=0 \mathrm{~K}$, only one-layer modification of $\mathrm{Nd}$ atoms affects the coercive field largely. 

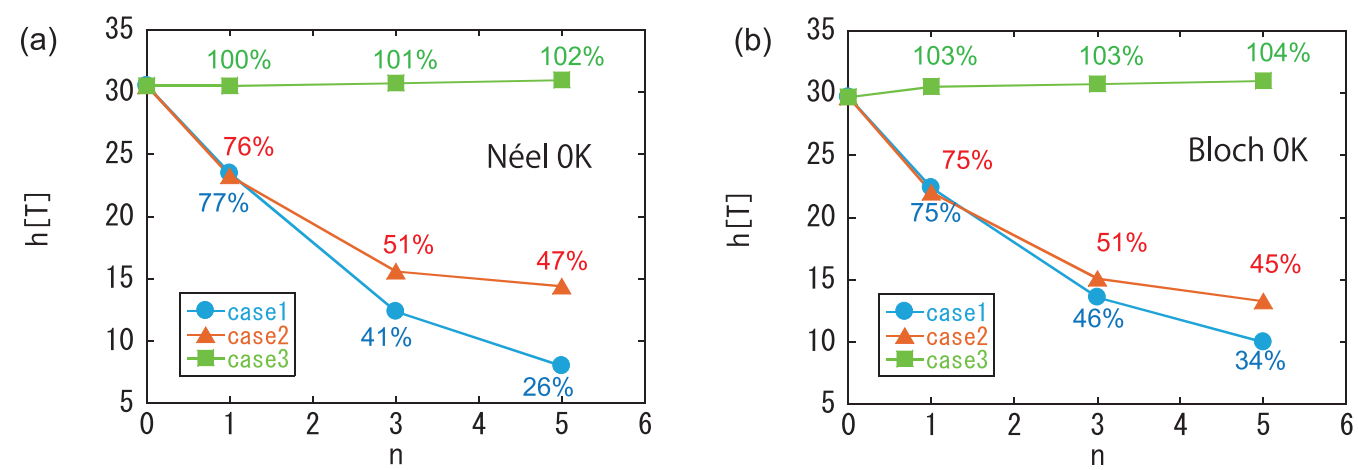

FIG. 5. $n$ dependence of threshold field at $0 \mathrm{~K}$ in cases $1-3$ for the (a) (001) and (b) (100) surfaces. The percentages are the ratios of the threshold fields for $n$ to those for $n=0$.

Note that the reduction in threshold field in case (1) (blue circles) is larger than that in case (2) (red triangles). This means that the in-plane anisotropy helps the coercivity in comparison with the case of no anisotropy. This tendency is different from that at finite temperatures studied in the next subsection. We consider the cause of this difference in the next subsection.

The threshold field in case (1) for the (001) surface is smaller than that for the (100) surface $(n \geqslant 3)$. This may be due to weaker exchange interactions along the $c$ axis than those along the $a$ axis $[22,25]$. A similar tendency was also seen in the nucleation field from a soft magnet phase in our previous study, in which the nucleation field for the Néel DW is smaller than that for the Bloch DW [16].

In case (3), the threshold field changes with $n$, e.g., $2 \%$ for $n=5$ at the (001) surface and $4 \%$ for $n=5$ at the (100) surface. This small increment is different from the dependence at finite temperatures. It is worth noting that for $n=$ 0 , the threshold fields are very high in both cases of the (001) and (100) surfaces. It is considered that for $n=0$, the magnetization reversal occurs in the Stoner-Wohlfarth mechanism in which all magnetic moments rotate simultaneously. Therefore, even if the surface anisotropy is reinforced, the reversal occurs in the bulk and the reinforcement effect is small.

\section{B. Finite-temperature properties}

In Fig. 6, we show $n$ dependence of the threshold field in cases (1)-(3) for the (001) surface at (a) $T=0.34 T_{\mathrm{c}}$, (c) $T=0.46 T_{\mathrm{c}}$, and (e) $T=0.69 T_{\mathrm{c}}$, and that for the (100) surface (b) at $T=0.34 T_{\mathrm{c}}$, (d) $T=0.46 T_{\mathrm{c}}$, and (f) $T=0.69 T_{\mathrm{c}}$. In contrast to the threshold field at $0 \mathrm{~K}$, one-layer modification for $\mathrm{Nd}$ atoms $(n=1)$ slightly affects the threshold fields at these finite temperatures in all the three cases [(1)-(3)] for both surfaces. For example, at $T=0.46 T_{\mathrm{c}}$, which is close to room temperature, the values of the threshold fields for one-layer modification are higher than $90 \%$ of those for $n=0$ for the (001) and (100) surfaces in cases (1) and (2). This result indicates that the thermal fluctuation smears out the effect of the surface in the case $n=1$.

However, the modification exerts relevant effects on the threshold field as $n$ increases. As the temperature increases, the threshold field for $n=0$ decreases and the effect of the modification becomes weaker because the thermal fluctuation becomes stronger and further smears the surface effect.

In contrast to the observation at $T=0 \mathrm{~K}$, we find that the threshold fields in case (2) are smaller than those in case (1) at finite temperatures, which indicates that the in-plane anisotropy accelerates magnetization reversal with the thermal fluctuation. We also confirmed this property at $T=0 \mathrm{~K}$ in a simplified system of an open chain, in which $\mathrm{Nd}$ atoms are connected through an exchange interaction, and only a few $\mathrm{Nd}$ atoms at one end have the anisotropy potential of case (1) or (2), and the others have the anisotropy in the bulk. We performed a calculation to find the minimum energy state, including the metastable state at a given field, and estimated the threshold field for magnetization reversal. The result shows that in a wide region of the strength of the exchange interaction, the coercive force in case (2) is larger than that in case (1). This property is interpreted as follows.

The minimum point of the anisotropy potential energy of the $\mathrm{Nd}$ atoms in the bulk is located at $\theta \simeq 0.2 \pi$ from the $c$ axis, as shown in Fig. 2 (blue line). On the other hand, for the $\mathrm{Nd}$ atoms in the modified layers in case (2), the anisotropy potential energy has a minimum at $\theta=\pi / 2$ [Fig. 2 (red line)]. Around the border between the modified and unmodified layers, the Nd magnetic moments in the modified layers indicate a certain $\theta$ smaller than $\theta=\pi / 2$ due to the effect from the bulk, whereas those in the unmodified layers indicate a certain $\theta$ larger than $\theta \simeq 0.2 \pi$ due to the effect from the modified $\mathrm{Nd}$ atoms. This compromise reduces the loss of the exchange energies and leads to a more stabilized configuration in case (2) than that in case (1), as shown by the threshold fields in Fig. 5.

In contrast to zero temperature, we find in Fig. 6 that case (1) is more stable than case (2). At finite temperatures, the effect of the thermal fluctuation plays an important role in the magnetization reversal. The anisotropy of the $\mathrm{Nd}$ atoms in the bulk is parallel to the $c$ axis at finite temperatures, and thus the in-plane anisotropy causes a large thermal fluctuation of magnetizations owing to the frustrated situation. This fluctuation causes the $\mathrm{Fe}$ moments in the modified layers and also in the vicinity of the modified layers to fluctuate markedly. This results in the acceleration of the magnetization reversal. In case (3), in contrast to the zero-temperature case, the threshold fields at finite temperatures increase for larger $n$, which is 

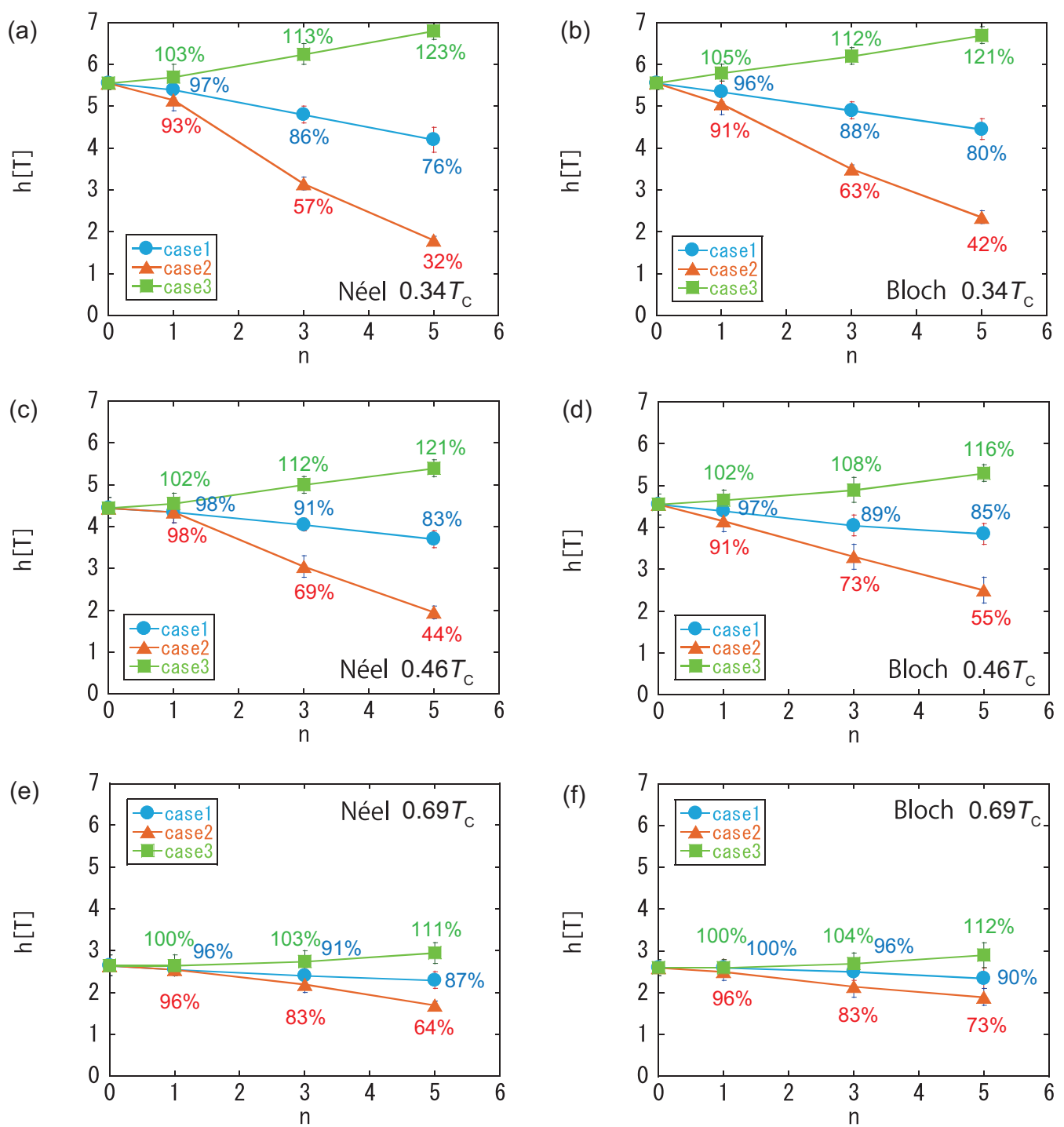

FIG. 6. $n$ dependence of the threshold field in cases (1)-(3) for the (001) surface at (a) $T=0.34 T_{\mathrm{c}}$, (c) $T=0.46 T_{\mathrm{c}}$, and (e) $T=0.69 T_{\mathrm{c}}$ and the (100) surface at (b) $T=0.34 T_{\mathrm{c}}$, (d) $T=0.46 T_{\mathrm{c}}$, and (f) $T=0.69 T_{\mathrm{c}}$. The percentages are the ratios of the threshold fields for $n$ to those for $n=0$.

naturally caused by the reinforcement effect of the anisotropy parallel to the $c$ axis.

In all three cases, the effect of the modification of the surface $\mathrm{Nd}$ atoms is larger for the (001) surface than for the (100) surface. This is probably because of the exchange interactions along the $c$ axis being weaker than those along the $a(b)$ axis, and thus the modification effect of the anisotropy energy for the (001) surface appears relatively stronger than that for the (100) surface.

\section{CONFIGURATIONS IN THE REVERSAL PROCESS}

To understand the feature of the domain growth in the magnetization reversal, we analyze snapshots of magnetic configurations. We show in Fig. 7 snapshots of the configuration of the magnetic moments in the system with the (001) surface at $T=0.34 T_{\text {c }}$ for (a) $n=0$ at its threshold field $h=5.5 \mathrm{~T}$ and (b) $n=5$ in case (2) at its threshold field $h=1.8 \mathrm{~T}$ (see also animation_001_a and animation_001_b in the Supplemental Material for detailed features [46]). The left panel is a snapshot of the start of magnetization reversal, the middle panel is that of domain wall propagation, and the right panel is that of the last stage of the reversal. In the case $n=0$, before magnetization reversal, a small up-spin domain appears on the right side around the surface in the left panel of Fig. 7(a). Similarly, small up-spin domains appear and disappear around the surface (see also animation_001_a in the Supplemental Material). Then, nucleation occurs near the surface and a domain grows along the $a$ and $b$ axes rapidly. The domain moves parallel to the $c$ axis. On the other hand, for $n=5$ before the start of the magnetization reversal, not only the $\mathrm{Nd}$ magnetic moments but also part of the Fe magnetic moments located between the first and fifth layers fluctuate to a much greater extent than those in the bulk because the $\mathrm{Fe}$ moments are affected by the fluctuation of the surface $\mathrm{Nd}$ moments through the exchange interactions between the $\mathrm{Nd}$ and $\mathrm{Fe}$ atoms. Thus, the domain forms more easily and grows parallel to the $c$ axis. Note that the border between the up- and 
(a)
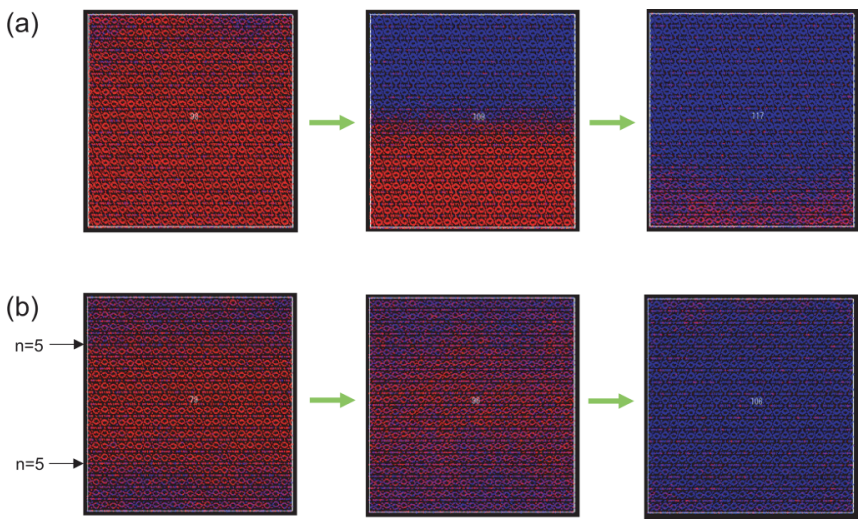

FIG. 7. Snapshots of the magnetic moments for the (001) surface at $T=0.34 T_{\mathrm{C}}$ for (a) $n=0$ at $h=5.5 \mathrm{~T}$ and (b) $n=5$ at $h=1.8 \mathrm{~T}$. The left panel of each figure is a snapshot of the start of magnetization reversal, the middle panel is that in the middle of the reversal, and the right panel is that of the last stage of the reversal. Red and blue denotes down-spin $\left(S_{i}^{z}<0\right)$ and up-spin states $\left(S_{i}^{z}>0\right)$, respectively.

down-spin parts is more unclear in Fig. 7(b) than in Fig. 7(a), although the external field is weaker. This is probably because the $\mathrm{Fe}$ moments in the bulk layers also fluctuate through strong exchange interactions.

We also show in Fig. 8 snapshots of the magnetic moments for the (100) surface at $T=0.34 T_{\mathrm{c}}$ for (a) $n=0$ at its threshold field $h=5.5 \mathrm{~T}$ and (b) $n=5$ in case (2) at its threshold field $h=2.3 \mathrm{~T}$ (see also animation_100_a and animation_100_b in the Supplemental Material for detailed features). Before the magnetization reversal for $n=0$, up-spin domains appear and disappear around the surface similarly to those observed in the (001) surface, but the domains are larger and the fluctuation range is larger (see also animation_001_b in the Supplemental Material). This is partially because the width of the Bloch DW is larger than that of the Néel DW [22]. After nucleation, the domain moves parallel to the $a$ axis. For $n=5$, the Nd moments and part of the Fe moments

(a)
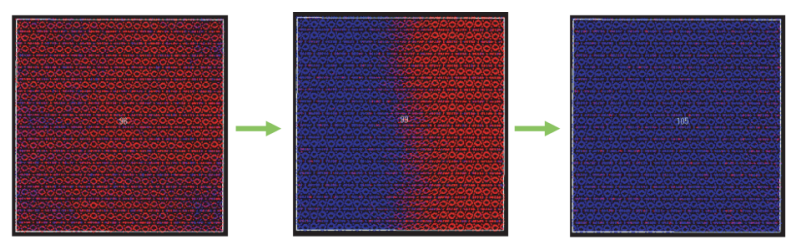

(b)
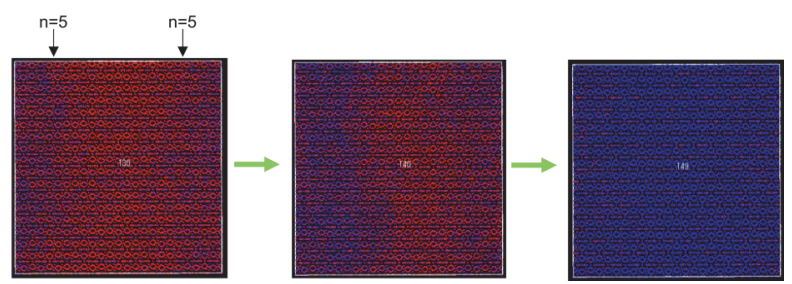

FIG. 8. Snapshots of the magnetic moments for the (100) surface at $T=0.34 T_{\mathrm{C}}$ for (a) $n=0$ at $h=5.5 \mathrm{~T}$ and (b) $n=5$ at $h=2.3 \mathrm{~T}$. The left panel of each figure is a snapshot of the start of magnetization reversal, the middle panel is that in the middle of the reversal, and the right panel is that of the last stage of the reversal. near the surface located up to around the fifth layer fluctuate to a greater extent than those in the bulk, but the range of the fluctuations is not so clear as that for the (001) surface. This is probably because a layered structure exists along the $c$ axis and also the DW width is larger than that in the case of the (001) surface. Similarly in Fig. 7, the border between the up- and down-spin parts is more unclear in Fig. 8(b) than in Fig. 8(a).

\section{SUMMARY}

We investigated how the modification of magnetic anisotropy of the Nd atoms located near the surface affects the coercivity at zero and finite temperatures by applying the stochastic LLG equation to the atomistic model. We studied the two types of surface, i.e., (001) and the (100) surfaces. We examined the three cases of modification, i.e., the $\mathrm{Nd}$ atoms in surface layers have (1) no anisotropy, (2) in-plane anisotropy, and (3) doubly reinforced anisotropy. We showed the temperature and modified-layer-depth dependences of the coercivity.

At zero temperature $(T=0 \mathrm{~K})$, one-layer modifications of the anisotropy of the $\mathrm{Nd}$ atoms in cases (1) and (2) largely reduce the coercivity. The reduction in case (1) is larger than that in case (2), in which the in-plane anisotropy helps to maintain the coercivity compared with no anisotropy. The reinforcement slightly affects the coercivity at $T=0 \mathrm{~K}$. In contrast to $T=0 \mathrm{~K}$, at finite temperatures above the SR transition point, the one-layer modifications hardly affect the coercive field in all three cases. This revises the result of $T=0 \mathrm{~K}$. Namely, the thermal fluctuation effect plays an important role in the magnetization reversal at finite temperatures.

As the number of modified layers, $n$, increases, the effect of modification becomes large. Unlike the zero-temperature case, at finite temperatures, the reduction in coercivity in case (1) is smaller than in case (2). Namely, the in-plane anisotropy suppresses the reversal at $T=0 \mathrm{~K}$ to a greater extent than the no anisotropy case, whereas it accelerates the reversal at finite temperatures. The difference is attributed to the change in the structure of the anisotropy potential energy between zero (the ground state) and finite temperatures above the SR transition point.

The coercivity increases significantly in case (3) at finite temperatures, which indicates that the surface treatment with strong anisotropic atoms would help the coercivity to increase. Unlike this situation, the coercivity slightly changes at $T=0 \mathrm{~K}$ in case (3), which is due to the uniform rotation. In all the three cases at finite temperatures, the effect of the modification of the surface $\mathrm{Nd}$ atoms is larger for the (001) surface than for the (100) surface for larger $n$, which is probably due to weaker effective exchange interactions along the $c$ axis than along the a (b) axis.

We also investigated features of the domain formation and propagation. As to the (001) surface without modification at finite temperatures, nucleation occurs suddenly near the surface and a domain grows along the $a$ and $b$ axes rapidly and a domain wall moves along the $c$ axis. On the other hand, for $n$ layer modification in case (2), the magnetic moments 
of not only the $\mathrm{Nd}$ atoms but also the Fe atoms located up to $n$ surface layer fluctuate largely before the magnetization reversal, which makes nucleation occur more easily. In the case of the (100) surface, a similar feature is observed, but magnetic domains fluctuate in a wider range regardless of the $n$ layer modification, which may be partially due to the larger domain-wall width along the $a(b)$ axis.

Our present study may provide useful detailed information to related experiments. The effect of the replacement of surface $\mathrm{Nd}$ atoms by Dy atoms on the coercivity is a challenging future subject, which requires atomistic information of the magnetic parameters of the replacement by Dy atoms. This will be studied in the future.

\section{ACKNOWLEDGMENTS}

The authors would like to thank Dr. Hirosawa for instructive discussion from experimental viewpoints, and Dr. Toga and Dr. Hinokihara for useful discussion from theoretical aspects. The present work was supported by the Elements Strategy Initiative Center for Magnetic Materials (ESICMM) (Grant No. 12016013) funded by the Ministry of Education, Culture, Sports, Science and Technology (MEXT) of Japan, and was partially supported by Grants-in-Aid for Scientific Research C (No. 18K03444 and No. 20K03809) from MEXT. The numerical calculations were performed on the Numerical Materials Simulator at the National Institute for Materials Science.
[1] S. Sugimoto, J. Phys. D 44, 064001 (2011)

[2] M. Sagawa and S. Hirosawa, J. Mater. Res. 3, 45 (1988).

[3] J. F. Herbst, J. J. Croat, F. E. Pinkerton, and W. B. Yelon, Phys. Rev. B 29, 4176 (1984).

[4] S. Hirosawa, Y. Matsuura, H. Yamamoto, S. Fujimura, M. Sagawa, and H. Yamauchi, J. Appl. Phys. 24, L803 (1985).

[5] A. V. Andreev, A. V. Deryagin, N. V. Kudrevatykh, N. V. Mushnikov, V. A. Reimer, and S. V. Terent'ev, Zh. Eksp. Teor. Fiz. 90, 1042 (1986) [Sov. Phys. JETP 63, 608 (1986)].

[6] H. Kronmüller and K.-D. Durst, J. Magn. Magn. Mater. 74, 291 (1988)

[7] J. F. Herbst, Rev. Mod. Phys. 63, 819 (1991).

[8] S. Hirosawa, Y. Matsuura, H. Yamamoto, S. Fujimura, M. Sagawa, and H. Yamauchi, J. Appl. Phys. 59, 873 (1986).

[9] O. Yamada, Y. Ohtsu, F. Ono, M. Sagawa, and S. Hirosawa, J. Magn. Magn. Mater. 70, 322 (1987).

[10] N. V. Mushnikov, P. B. Terent'ev, and E. V. Rosenfel'd, Phys. Met. Metallogr. 103, 39 (2007).

[11] R. Friedberg and D. I. Paul, Phys. Rev. Lett. 34, 1234 (1975).

[12] A. Sakuma, S. Tanigawa, and M. Tokunaga, J. Magn. Magn. Mater. 84, 52 (1990).

[13] A. Sakuma, J. Magn. Magn. Mater. 88, 369 (1990).

[14] S. Mohakud, S. Andraus, M. Nishino, A. Sakuma, and S. Miyashita, Phys. Rev. B 94, 054430 (2016).

[15] A. L. Wysocki and V. P. Antropov, J. Magn. Magn. Mater. 428, 274 (2017).

[16] I. E. Uysal, M. Nishino, and S. Miyashita, Phys. Rev. B 101, 094421 (2020).

[17] S. Okamoto, R. Goto, N. Kikuchi, O. Kitakami, T. Akiya, H. Sepehri-Amin, T. Ohkubo, K. Hono, K. Hioki, and A. Hattori, J. Appl. Phys. 118, 223903 (2015).

[18] T. Pramanik, A. Roy, R. Dey, A. Rai, S. Guchhait, H. C. P. Movva, C.-C. Hsieh, and S. K. Banerjee, J. Magn. Magn. Mater. 437, 72 (2017).

[19] S. Hirosawa, M. Nishino, and S. Miyashita, Adv. Nat. Sci.: Nanosci. Nanotechnol. 8, 013002 (2017).

[20] H. Kronmüllar and M. Fähnle, 'Micromagnetism and the Microstructure of Ferromagnetic Solids (Cambridge University Press, Cambridge, 2003).

[21] Y. Toga, M. Matsumoto, S. Miyashita, H. Akai, S. Doi, T. Miyake, and A. Sakuma, Phys. Rev. B 94, 174433 (2016).
[22] M. Nishino, Y. Toga, S. Miyashita, H. Akai, A. Sakuma, and S. Hirosawa, Phys. Rev. B 95, 094429 (2017).

[23] T. Hinokihara, M. Nishino, Y. Toga, and S. Miyashita, Phys. Rev. B 97, 104427 (2018).

[24] S. Miyashita, M. Nishino, Y. Toga, T. Hinokihara, T. Miyake, S. Hirosawa, and A. Sakuma, Scr. Mater. 154, 259 (2018).

[25] Y. Toga, M. Nishino, S. Miyashita, T. Miyake, and A. Sakuma, Phys. Rev. B 98, 054418 (2018).

[26] M. Nishino and S. Miyashita, Phys. Rev. B 100, 020403(R) (2019).

[27] Y. Toga, S. Miyashita, A. Sakuma, and T. Miyake, npj Comput. Mater. 6, 67 (2020).

[28] M. Nishino, I. E. Uysal, T. Hinokihara, and S. Miyashita, Phys. Rev. B 102, 020413(R) (2020).

[29] Q. Gong, M. Yi, R. F. L. Evans, B.-X. Xu, and O. Gutfleisch, Phys. Rev. B 99, 214409 (2019).

[30] Q. Gong, M. Yi, and B.-X. Xu, Phys. Rev. Materials 3, 084406 (2019)

[31] Q. Gong, M. Yi, R. F. L. Evans, O. Gutfleisch, and B.-X. Xu, Mater. Res. Lett. 8, 89 (2020).

[32] S. C. Westmoreland, R. F. L. Evans, G. Hrkac, T. Schrel, G. T. Zimanyi, M. Winklhofer, N. Sakuma, M. Yano, A. Kato, T. Shoji, A. Manabe, M. Ito, and R. W. Chantrell, Scr. Mater. 148, 56 (2018).

[33] S. C. Westmoreland, C. Skelland, T. Shoji, M. Yano, A. Kato, M. Ito, G. Hrkac, T. Schrefl, R. F. L. Evans, and R. W. Chantrell, J. Appl. Phys. 127, 133901 (2020).

[34] Y. Tatetsu, S. Tsuneyuki, and Y. Gohda, Phys. Rev. Appl. 6, 064029 (2016).

[35] Y. Ainai, Y. Tatetsu, A. Terasawa, and Y. Gohda, Appl. Phys. Express 13, 017006 (2020).

[36] M. Yamada, H. Kato, H. Yamamoto, and Y. Nakagawa, Phys. Rev. B 38, 620 (1988).

[37] H. Moriya, H. Tsuchiura, and A. Sakuma, J. Appl. Phys. 105, 07A740 (2009).

[38] S. Tanaka, H. Moriya, H. Tsuchiura, A. Sakuma, M. Diviš, and P. Novák, J. Appl. Phys. 109, 07A702 (2011).

[39] C. Mitsumata, H. Tsuchiura, and A. Sakuma, Appl. Phys. Express 4, 113002 (2011).

[40] J. L. García-Palacios and F. J. Lázaro, Phys. Rev. B 58, 14937 (1998). 
[41] M. Nishino and S. Miyashita, Phys. Rev. B 91, 134411 (2015).

[42] T.-H. Kim, T. T. Sasaki, T. Ohkubo, Y. Takada, A. Kato, Y. Kaneko, and K. Hono, Acta Mater. 172, 139 (2019).

[43] A. I. Liechtenstein, M. I. Katsnelson, V. P. Antropov, and V. A. Gubanov, J. Magn. Magn. Mater. 67, 65 (1987).
[44] Y. Miura, H. Tsuchiura, and T. Yoshioka, J. Appl. Phys. 115, 17A765 (2014).

[45] A. J. Freeman and R. E. Watson, Phys. Rev. 127, 2058 (1962).

[46] See Supplemental Material at http://link.aps.org/supplemental/ 10.1103/PhysRevB.103.014418 for movies showing magnetization reversal in Figs. 7 and 8. 